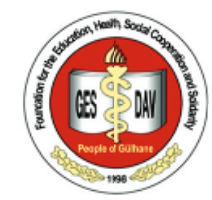

GESDAV

\title{
Clustering of behavioral risk factors in the Portuguese population: Data from National Health Interview Survey
}

\author{
Elsa Costa ${ }^{1}$, Carlos Matias Dias ${ }^{1,4}$, Luísa Oliveira², Luzia Gonçalves ${ }^{3}$
}

${ }^{1}$ Departamento de Estratégias de Acção em Saúde, Escola Nacional de Saúde Pública/ Universidade Nova de Lisboa, Avenida Padre Cruz, 1600-560 Lisboa, Portugal, Europe, 2Departamento de Alimentação e Nutrição, Instituto Nacional de Saúde Doutor Ricardo Jorge, I.P., Avenida Padre Cruz, 1649-016 Lisboa, Portugal, Europe, ${ }^{3}$ Unidade de Saúde Pública Internacional e Bioestatística, Instituto de Higiene e Medicina Tropical, Universidade Nova de Lisboa, Rua da Junqueira, 100, 1349-008 Lisboa, Portugal and CEAUL, Europe, ${ }^{4}$ Departamento de Epidemiologia, Instituto Nacional de Saúde Doutor Ricardo Jorge, I.P., Avenida Padre Cruz, 1649-016 Lisboa, Portugal, Europe

Address for correspondence: Elsa Costa, Departamento de Estratégias de Acção em Saúde, Escola Nacional de Saúde Pública/Universidade Nova de Lisboa, Avenida Padre Cruz, 1600-560 Lisboa, Portugal, Europe. E-mail: elsa.costa@ensp. unl.pt

Received: August 30, 2014 Accepted: November 22, 2014 Published: December 06, 2014

\begin{abstract}
Background: Given that behavior-related risk factors cluster together in individuals and populations it is important to study their patterns to inform Public Health interventions and decisions aimed at controlling disease and promoting health. This study examines the clustering and variation across different socio-demographic groups of four major behavioral risk factors (smoking, heavy drinking, physical inactivity, and unhealthy diet) in two groups of the Portuguese adult population, one with and one without diabetes. Methods: The study sample was derived from the 2005/2006 National Health Interview Survey for Portugal. Clustering was evaluated by comparing the observed and expected frequency of the different possible combinations of the four risk factors. A binary multiple logistic regression model was fitted to examine the socio-demographic variation in the clustering of the four risk factors. Results: Among the Portuguese population $(584,286$ individuals surveyed), $8.9 \%$ of diabetics and $19.5 \%$ of non-diabetics accumulated two or three behavioral risk factors. Behavioral risk factors are explored considering all possible multiple combinations $(k=16)$. The most frequent combination was smoking and unhealthy diet. Conclusions: These findings suggest that the likelihood of individuals having two or more risk behaviors simultaneously was greater in men 35-44 years old and lower education level both in diabetics and non-diabetics.
\end{abstract}

KEY WORDS: Behavioral risk factors, diabetes, diet, drinking, smoking 


\section{INTRODUCTION}

Changes in human behavior and lifestyle over the last century resulted in a dramatic increase of the incidence of diabetes worldwide [1]. Increases in the prevalence of smoking, heavy drinking, an unhealthy diet, and physical inactivity are the principal modifiable causes of non-communicable morbidity and mortality [2-4].

There is vast epidemiological evidence that indicates these four behavioral risk factors contribute to the development of chronic conditions, such as different types of cancer, Type-2 diabetes, and cardiovascular disease [3-5]. While much is known about each one of the behavioral-related risk factors, less is known about the prevalence and clustering of multiple risk factors in representative samples of the population $[2,6]$.

Smoking, excessive alcohol use, an unhealthy diet, and physical inactivity have been shown to be more prevalent among men, younger age groups, economically inactives, single status, and those who have a lower socio-economic status and lower level of education $[7,8]$. There is some evidence that combinations of behavioral risk factors has important implications on people's health $[3,9]$, suggesting that the clustering of lifestyle risk factors are more harmful than can be expected from the individual effects alone.

Insight into clustering of lifestyle risk factors is important because this can be used in developing prevention strategies by targeting groups in the population with a simultaneous presence of lifestyle-related unhealthy habits [4]. We defined clustering as an observed proportion of a combination of risk factors in excess of its expected proportion [10]. In this context, it is important to know if we can discriminate subgroups with elevated clustering so that prevention can be better targeted and organized [10]. Hence, the study of the clustering of risk factors has important implications on both disease risk and the development of preventive interventions targeting the combination of risk factors rather than individual risk factors [3].

In the present study the prevalence and clustering of lifestyle risk factors were investigated in the Portuguese population, diabetics and non-diabetics, aged 15 years and over, in total and in subgroups based on the sex, age, marital status and education level.

The aim of the present study was to explore the clustering of four major behavioral risk factors in two subgroups of the Portuguese population: With diabetes and without diabetes. The focus is physical inactivity, unhealthy diet, excessive alcohol, and smoking, as these are the main modifiable causes of ill health in the developed world $[11,12]$. In addition, this study explores the socio-demographic variation in the clustering of the four behavioral risk factors in order to identify the groups that are the most at risk.

\section{METHODS}

\section{Study Population}

The study population was the Portuguese population aged 15 years and over living in private households through a representative sample associated to Portuguese National Health Interview Survey [13]conducted between February 2005 and February 2006. The sampling frame was selected from the nationally representative sample of all housing units in the five administrative regions (North, Centre, Lisbon region, Alentejo and Algarve)and the two autonomous regions of Azores and Madeira. The population living in collective households and other non-classical households (e.g. hospitals, prisons, military barracks, or retirement houses)were not included. Data on participants younger than 15 years were excluded from this analysis because of the low prevalence of diabetes in this subgroup [13]. Cases with missing data were also removed from the analysis. This study evaluated the population surveyed in the second trimester of data collection because physical activity of respondents was only assessed in this trimester.

The data collection process was conducted using probabilistic sampling methods of the Portuguese population, through interviews at home, using valid and stable instruments and procedures. A description of the methodology of sample selection is published [13]. Self-reported data from diabetic and non-diabetic individuals, hereinafter referred to as diabetic/ non-diabetic, were then analyzed.

\section{Measures}

\section{Behavioral Risk Factors}

Four behavioral risk factors were studied [Table 1]. People were asked "Do you smoke?." People answering "no" were considered nonsmokers, and those who answered "daily" or "occasionally," smokers.

Definition of alcohol consumption was based on the centers for disease control and prevention definition of heavy drinking as consuming an average of more than two drinks for men and one drink or more for women, per day [14]. A standard drink was that containing $10 \mathrm{~g}$ of alcohol, which in Portugal is a glass of beer, a glass of wine or a measure of distilled alcohol beverage [15]. Those respondents who stated they drunk during the previous week were asked questions about the average number of glasses of alcoholic beverages (including wine, beer, brandy, spirits, whisky/gin/vodka)drunk per day and the mean volume of each serving (for each type of alcoholic beverage)was assessed using visual aids. Daily alcohol consumption was assessed by average number of servings per day $\times$ mean volume of each serving $\times$ mean $\%$ alcohol (12\% for wine, $5 \%$ for beer, $20 \%$ for liquor and $40 \%$ for spirits) $\times 0.8$ (alcohol density)for each type of alcoholic beverage [16]. Total alcohol consumption in the day was assessed by summing up the individual amounts for each type of alcoholic beverage. 
Table 1: Socio-demographic characteristics and unhealthy behavior risk factors of the Portuguese population aged 15 years and over with diabetes and without diabetes ${ }^{\mathrm{a}}$

\begin{tabular}{|c|c|c|c|c|}
\hline \multirow[t]{2}{*}{ Variable } & \multicolumn{2}{|c|}{$\begin{array}{c}\text { Diabetics } \\
(n=274,293)\end{array}$} & \multicolumn{2}{|c|}{$\begin{array}{l}\text { Non-diabetics } \\
(n=309,993)\end{array}$} \\
\hline & $n^{b}$ & $\%^{c}$ & $n^{b}$ & $\%^{c}$ \\
\hline \multicolumn{5}{|l|}{ Sex } \\
\hline Men & 107,078 & 39.0 & 132,516 & 42.7 \\
\hline Women & 167,215 & 61.0 & 177,478 & 57.3 \\
\hline \multicolumn{5}{|l|}{ Age } \\
\hline $15-34$ & 14,229 & 5.2 & 113,306 & 36.6 \\
\hline $35-44$ & 17,349 & 6.3 & 49,499 & 16.0 \\
\hline $45-54$ & 32,715 & 11.9 & 45,695 & 14.7 \\
\hline $55-64$ & 77,562 & 28.3 & 47,626 & 15.4 \\
\hline $65-74$ & 74,229 & 27.1 & 27,941 & 9.0 \\
\hline$\geq 75$ & 58,208 & 21.2 & 25,926 & 8.4 \\
\hline \multicolumn{5}{|l|}{ Marital status } \\
\hline Single & 18,657 & 6.8 & 97,473 & 31.4 \\
\hline Married & 188,276 & 68.6 & 174,183 & 56.2 \\
\hline Divorced & 10,152 & 3.7 & 11,937 & 3.9 \\
\hline Widower & 57,208 & 20.9 & 26,400 & 8.5 \\
\hline \multicolumn{5}{|l|}{ Education level } \\
\hline None & 76,952 & 28.1 & 41,114 & 13.3 \\
\hline Primary & 178,078 & 64.9 & 181,998 & 58.7 \\
\hline Secondary & 9,614 & 3.5 & 42,860 & 13.8 \\
\hline Higher & 9,649 & 3.5 & 44,022 & 14.2 \\
\hline \multicolumn{5}{|l|}{ Risk behaviors } \\
\hline Smoking & 19,329 & 7.0 & 63,350 & 20.5 \\
\hline Heavy drinking & 986 & 0.4 & 1,618 & 0.5 \\
\hline Physical inactivity & 8,109 & 19.6 & 7,243 & 10,1 \\
\hline Unhealthy diet & 249,502 & 91.8 & 274,082 & 89.2 \\
\hline \multicolumn{5}{|c|}{ Number of risk behaviors } \\
\hline None & 20,841 & 7.6 & 25,170 & 8.1 \\
\hline One & 229,141 & 83.5 & 225,468 & 72.7 \\
\hline Two & 24,149 & 8.8 & 57,239 & 18.5 \\
\hline Three & 162 & 0.1 & 2,116 & 0.7 \\
\hline
\end{tabular}

a2005/2006 Portuguese National Health Interview Survey, Trimestre 2, ${ }^{b}$ Weighted analysis expressed in terms of absolute frequency of Portuguese population aged 15 years and over, "Weighted analysis expressed in terms of percentage of Portuguese population aged 15 years and over, ${ }^{\text {T}}$ The study population does not have the simultaneous presence of four behavioral risk factors

The third behavioral risk factor was physical inactivity. The respondents were asked about which was the time usually spent in l day during the previous week doing vigorous physical activities (e.g. heavy work, aerobics, running, swimming, or anything else that causes large increases in breathing or heart rate)and moderate physical activities (e.g. bicycling, vacuuming, gardening). Respondents were classified as being moderately physically active if they reported engaging in moderate intensity activity at least $30 \mathrm{~min} /$ day or vigorously physically active if they reported engaging in vigorous-intensity activity at least $20 \mathrm{~min} /$ day $[17,18]$. Hence, the practice of $<30 \mathrm{~min}$ of moderate physical activity per day or the practice of $<20$ minutes of vigorous physical activity per day was considered a risk factor.

The fourth behavioral risk factor focused on the unhealthy diet. The unhealthy diet was assessed in a previous study [19] that explored the unhealthy dietary pattern using current nutrition knowledge and latent class analysis. With respect to unhealthy dietary pattern, we reported dietary nondiversity, nonconsumption of fruit and vegetables, and number of main meals per day below three as indicators of an unhealthy diet.

\section{Socio-demographic Variables}

The socio-demographic characteristics: Sex, age, marital status, and level of education were included in this study [Table 1]. Age was categorized as 15-34 years, 35-44 years, 45-54 years, 55-64 years, 65-74 years, and 375 years intervals; marital status was classified into single, married, divorced, and widowed. Level of education was divided into four categories: None, primary, secondary, and higher.

\section{Statistical Analyses}

We described the observed frequency of single behavioral risk factors coded as a binary variable (yes $=1$; no $=0$ ). Then the observed frequency of multiple behavioral risk factors: Smoking, heavy drinking, physical inactivity, and unhealthy diet were estimated using a risk factor index approach where individual risk factor scores were summed to yield a multiple risk factor index ranging from zero (no risk behavior)to four (four simultaneous risk behavioral) based on the overall factors. To evaluate the most frequent risk behavioral combinations, the ratio between the observed and expected $(\mathrm{O} / \mathrm{E})$ frequency was calculated for each possible combination, as described by Schuit et al. (2002). The expected frequency was calculated by multiplying the individual probabilities of each risk factor, assuming risk factors occur independently in the population under study [10]. Clustering occurs when the observed prevalence of a combination of factors exceeds the expected prevalence for this combination. $\mathrm{O} / \mathrm{E}$ ratios higher than 1 are indicative of clustering [4]. All analyses were conducted for diabetics and non-diabetics separately.

In the first analysis [Table 3] the crude odds ratio (OR) was calculated using the clustering of two behaviors in the presence of another risk behavioral. Reference category is those not exposed to the first risk factor. For example, an OR of 6.492 indicates that subjects displaying a given behavioral (e.g., unhealthy diet)are 6.5 times more likely to display another behavior (e.g. physical inactivity)when compared to those not exposed to the first behavior (unhealthy diet).

A binary multiple logistic regression model was carried out with presence of a set of the behavior risk factors as the dependent variable: A respondent has at least two behavioral risk factors as compared to the reference group of having zero behavior risk factor and the socio-demographic characteristics as covariates: Sex, age, marital status and education level, in order to obtain adjusted $\mathrm{OR}_{\mathrm{adj}}$ and their corresponding 95\% confidence intervals. In addition, we interpreted the magnitude of the association between the different socio-demographic variables and the presence of the "worst" combinations (at least two behavior risk factors). 
Table 2: Risk behavior clusters of the Portuguese population aged 15 years and over with diabetes and without diabetes

\begin{tabular}{|c|c|c|c|c|c|c|c|c|c|c|c|}
\hline \multirow{2}{*}{$\begin{array}{l}\text { Number of } \\
\text { risk factors }\end{array}$} & \multicolumn{4}{|c|}{ Presence of risk behaviors } & & \multicolumn{3}{|c|}{ Diabetics $(n=274,293)$} & \multicolumn{3}{|c|}{ Non-diabetics $(n=309,993)$} \\
\hline & Smoking & Heavy drinking & Physical inactivity & Unhealthy diet & & $0(\%)$ & $E(\%)^{a}$ & 0/E (Approx.) & $0(\%)$ & $E(\%)^{a}$ & 0/E (Approx.) \\
\hline \multirow[t]{2}{*}{4} & + & + & + & + & & 0.0 & 0.0 & 0.0 & 0.0 & 0.0 & 0.0 \\
\hline & & & & & Total & 0.0 & 0.0 & 0.0 & 0.0 & 0.0 & 0.0 \\
\hline \multirow[t]{5}{*}{3} & + & + & + & - & & 0.0 & 0.0 & 0.0 & 0.0 & 0.0 & 0.0 \\
\hline & + & + & - & + & & 0.1 & 0.0 & 4.8 & 0.0 & 0.1 & 0.0 \\
\hline & + & - & + & + & & 0.0 & 1.3 & 0.0 & 0.3 & 1.8 & 0.2 \\
\hline & - & + & + & + & & 0.0 & 0.1 & 0.0 & 0.0 & 0.0 & 0.0 \\
\hline & & & & & Total & 0.1 & 1.3 & 0.1 & 0.3 & 2.0 & 0.2 \\
\hline \multirow[t]{7}{*}{2} & + & + & - & - & & 0.0 & 0.0 & 0.0 & 0.2 & 0.0 & 20.1 \\
\hline & + & - & + & - & & 0.0 & 0.1 & 0.0 & 0.3 & 0.2 & 1.3 \\
\hline & + & - & - & + & & 5.8 & 5.1 & 1.1 & 11.5 & 16.4 & 0.7 \\
\hline & - & + & + & - & & 0.0 & 0.0 & 0.0 & 0.0 & 0.0 & 0.0 \\
\hline & - & + & - & + & & 0.1 & 0.3 & 0.4 & 0.0 & 0.3 & 0.0 \\
\hline & - & - & + & + & & 10.9 & 16.7 & 0.7 & 5.4 & 7.1 & 0.8 \\
\hline & & & & & Total & 16.8 & 22.2 & 0.8 & 17.4 & 24.0 & 0.7 \\
\hline \multirow[t]{5}{*}{1} & + & - & - & - & & 1.1 & 0.5 & 2.4 & 1.7 & 2.0 & 0.9 \\
\hline & - & + & - & - & & 0.2 & 0.0 & 8.2 & 0.1 & 0.0 & 2.6 \\
\hline & - & - & + & - & & 0.1 & 1.5 & 0.1 & 0.2 & 0.9 & 0.2 \\
\hline & - & - & - & + & & 82.9 & 68.4 & $1.2^{(*)}$ & 76.4 & 63.4 & $1.2^{(*)}$ \\
\hline & & & & & Total & 84.3 & 70.3 & 1.2 & 78.4 & 66.3 & 1.2 \\
\hline \multirow[t]{2}{*}{0} & - & - & - & - & & 6.7 & 6.1 & 1.1 & 7.0 & 7.7 & 0.9 \\
\hline & & & & & Total & 6.7 & 6.1 & 1.1 & 7.0 & 7.7 & 0.9 \\
\hline
\end{tabular}

Notes: Prevalence was computed using weighted methods and expressed in terms of percentage of Portuguese population aged 15 years and over, $+:$ Presence of unhealthy behavior. -: Absence of unhealthy behavior. 0 : observed prevalence of combination of risk factors; E: expected prevalence of combination of risk factors; O/E: observed/expected, ${ }^{a} E(\%)$ : Some of them are very small but not zero, as suggested in same cases, (*) For example in both diabetics and non-diabetics the proportion of diabetics and non-diabetics having one risk behavior (unhealthy diet) was higher than can be expected on the basis of the individual frequencies (0/E ratio in diabetics: 1.2, non-diabetics: 1.2 ). This indicates a $20 \%$ increase in subjects with an unhealthy diet over that which would be expected if the risk factors were independent

Table 3: OR and 95\% CI of combination of two behavioral risk factors versus first factor in diabetics and non-diabetics

\begin{tabular}{|c|c|c|c|c|c|c|}
\hline & \multicolumn{3}{|c|}{ Diabetics $(n=274,293)$} & \multicolumn{3}{|c|}{ Non-diabetics $(n=309,993)$} \\
\hline & $\%$ & $\mathrm{OR}^{\mathrm{a}}$ & $95 \% \mathrm{CI}$ & $\%$ & $\mathrm{OR}^{\mathrm{a}}$ & $95 \% \mathrm{CI}$ \\
\hline \multicolumn{7}{|l|}{ Risk factor combination } \\
\hline Smoking $\times$ heavy drinking & 17.9 & 2.911 & $2.472-3.429$ & 61.8 & 10.040 & $9.273-10.869$ \\
\hline Smoking $\times$ unhealthy diet & 6.5 & 0.418 & $0.402-0.436$ & 13.3 & 0.515 & $0.504-0.526$ \\
\hline Smoking $\times$ physical inactivity & 0.0 & $\mathrm{Na}$ & $\mathrm{Na}$ & 23.0 & 1.356 & $1.301-1.413$ \\
\hline Heavy drinking $\times$ unhealthy diet & 0.1 & 0.048 & $0.042-0.054$ & 0.1 & 0.022 & $0.020-0.024$ \\
\hline Heavy drinking $\times$ physical inactivity & 0.0 & $\mathrm{Na}$ & $\mathrm{Na}$ & 0.0 & $\mathrm{Na}$ & $\mathrm{Na}$ \\
\hline Unhealthy diet $\times$ physical inactivity & 98.0 & 6.492 & $5.540-7.608$ & 84.3 & 1.032 & $0.985-1.082$ \\
\hline
\end{tabular}

${ }^{a}$ Reference category is those not exposed to the first risk factor, Na: Not applicable, OR: Odds ratios, CI: Confidence intervals

The data analysis procedures were carried out using the SPSS Statistical Software Package version 21.0 (IBM SPSS Statistics 20), considering the sampling weight.

\section{RESULTS}

Table 1 shows the socio-demographic characteristics of the sample and the frequency of each individual and studied unhealthy behavior in each group: Diabetics and non-diabetics. About $14 \%$ of the studied Portuguese population aged 15 years and over smoked, $0.4 \%$ drank heavily an average in the last week, $2.6 \%$ were physical inactive, and $89.6 \%$ had an unhealthy diet. While non-diabetics were more likely to have smoking as risk behavior, diabetics were more likely to have physical inactivity and unhealthy diet.
Analysis comparing diabetics and non-diabetics in the study population showed statistically significant differences between the two groups with respect to sex, age, marital status, and education level. The unhealthy diet was the most frequent single behavior, with a significant difference between diabetic and non-diabetic individuals (91.8\% vs. $89.2 \%, \mathrm{p}<0.001)$.

Table 2 presents the occurrence of the 16 possible combinations of the four unhealthy behaviors investigated. For the combination of two unhealthy behaviors, we noted an important difference between the combination of smoking and heavy drinking in nondiabetics $(\mathrm{O} / \mathrm{E}=20.1)$. For the combination of three unhealthy behaviors, the combination that presented the highest potential for aggregation was smoking, heavy drinking, and unhealthy diet $(\mathrm{O} / \mathrm{E}=4.8)$ for diabetics. Non-diabetics have a low aggregation 
of three behavior factors. The cluster of three or two unhealthy behaviors presented the greatest difference between $\mathrm{O} / \mathrm{E}$ proportions in diabetics and non-diabetics, respectively.

Clustering for pairs of unhealthy behaviors is presented in Table 3. It is clear that the risk of lacking a healthy diet is markedly higher for individuals who have physical inactivity, among diabetics $(\mathrm{OR}=6.5)$ and the risk of smoking is higher for individuals who have an excessive alcohol consumption, among non-diabetics $(\mathrm{OR}=10.0)$. Furthermore, diabetics and non-diabetics individuals who have a healthy diet are more likely to smoke, as well as to drink.

Table 4 presents the results of the binary logistic model with a dichotomization of behavior risk factors (zero vs. two or more) as the dependent variable. This table shows the association between socio-demographic variables and presence of at least two behavior factors (prevalence $=8.9 \%$ diabetics; prevalence $=19.2 \%$ non-diabetics). Men aged 35-44 years with secondary education level were more likely to have at least two risk factors, in both diabetics and non-diabetic individuals, taking into account the reference categories as indicated in Table 4.

\section{DISCUSSION}

In the present study, we investigated the frequency and clustering of the four most important behavioral risk factors for the development and control of diabetes, namely smoking, heavy drinking, physical inactivity and unhealthy diet. Our results show that approximately $14 \%$ of the Portuguese population aged 15 years and over had at least two behavioral risk factors simultaneously.

In recent years, a number of studies reported clustering of different behavioral risk factors $[2,4,8]$. However, it is difficult to

Table 4: Association between socio-demographic variables and presence of two or more risk behavioral factors, among diabetic and non-diabetic individuals

\begin{tabular}{|c|c|c|c|c|}
\hline \multirow{2}{*}{$\begin{array}{l}\text { Socio-demographic } \\
\text { variables }\end{array}$} & \multicolumn{2}{|c|}{ Diabetics } & \multicolumn{2}{|c|}{ Non-diabetics } \\
\hline & OR ${ }_{\text {adj }}$ & $95 \% \mathrm{CI}^{\mathrm{a}}$ & $\mathrm{OR}_{\mathrm{adj}}$ & $95 \% \mathrm{CI}^{\mathrm{a}}$ \\
\hline \multicolumn{5}{|l|}{ Sex (Women) } \\
\hline Men & 2.98 & $2.83-3.14$ & 2.58 & $2.51-2.65$ \\
\hline \multicolumn{5}{|l|}{ Age $(\geq 75)$} \\
\hline $15-24$ & 0.88 & $0.78-0.98$ & 7.26 & $6.84-7.70$ \\
\hline $25-34$ & 1.62 & $1.47-1.79$ & 8.06 & $7.60-8.55$ \\
\hline $35-44$ & 12.92 & $11.66-14.32$ & 12.81 & $12.01-13.67$ \\
\hline $45-54$ & 4.22 & $3.87-4.60$ & 5.68 & $5.37-6.02$ \\
\hline $55-64$ & 1.66 & $1.54-1.79$ & 5.95 & $5.61-6.31$ \\
\hline \multicolumn{5}{|c|}{ Marital status (Married) } \\
\hline Single & 1.35 & $1.21-1.51$ & 0.62 & $0.59-0.64$ \\
\hline Divorced & 0.45 & $0.41-0.48$ & 1.33 & $1.26-1.39$ \\
\hline Widower & 0.50 & $0.46-0.54$ & 0.39 & $0.37-0.41$ \\
\hline \multicolumn{5}{|c|}{ Education level (Higher) } \\
\hline None & 10.02 & $8.92-11.26$ & 0.64 & $0.60-0.68$ \\
\hline Primary & 8.84 & $7.95-9.83$ & 0.72 & $0.68-0.75$ \\
\hline Secondary & 89.26 & $76.28-104.45$ & 1.51 & $1.43-1.59$ \\
\hline
\end{tabular}

Notes: The reference groups of predictor variables are given in

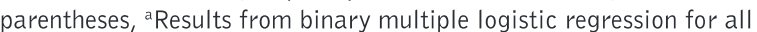
socio-demographic variables compare these studies as they focus on different combinations of behavioral risk factors, use different cut-off points, concern different study populations $[3,7,10]$.

In this study more people than expected had a combination of three behavioral risk factors including smoking, heavy drinking and unhealthy diet. This finding is consistent with the studies carried out in The Nederlands [10] and England [2].

The prevalence order of risk behaviors in our study with the diabetic population is similar to the findings from the Morgen study $[10]$ conducted with Dutch individuals aged $20-65$ years which showed that the most common health risk behavior was poor diet, followed by low levels of physical activity, smoking, and heavy drinking. A population-based survey derived from the 2003 Health Survey for England [2] showed the same pattern.

The results of this study show that certain combinations were less prevalent than could have been expected on the basis of the occurrence of the individual behavioral risk factors alone. These mainly involved unhealthy diet, together with smoking and/or physical inactivity, suggesting that people who have a healthy diet are more likely to smoke and/or to practice physical exercise. It has been hypothesized that this may be due to people smoking after participating in organized sports [10].

This study also found that the clustering of sets of behavioral risk factors was more marked for diabetics than for non-diabetics, particularly between physical inactivity and unhealthy diet. This finding suggests that more diabetics than expected had none of the four behaviors risk factors.

In a study conducted in Brazil [20]the combination of simultaneous risk factors for chronic noncommunicable diseases, with at least two factors present, was $59.4 \%$ of the respondents and the most frequent pattern was the simultaneity of inadequate diet and physical inactivity (30.6\%).

In addition, having the specific combination of smoking and unhealthy diet was clustered more strongly for non-diabetics than for diabetics. As there is no clear explanation for these results, the differences between diabetics and non-diabetics clustering should be more closely scrutinized.

Our results show that there are specific groups within the adult Portuguese population with diabetes and without diabetes that have an overall risky lifestyle. The results suggested that multiple risk factors were more prevalent among men who had 35-44 years and secondary education level. Men, in general, were more likely to report risk factors than women. Researchers who have addressed gender differences reported that socioeconomic and cultural factors can influence these behaviors [21].

The literature [10] report that the relationship between marital status and health could be related to a protective effect on the health status by the social and economic support between married people. In our study we found that single diabetic individuals and divorced non-diabetic individuals are more 
likely to present at least two risk behaviors simultaneously than married diabetic and non-diabetic individuals, respectively.

Lower levels of education were also strongly associated with the presence of two or more risk factors in diabetics. This social gradient was also observed in studies about the simultaneity of risk factors in England [2], Holland [10] and the United States [22].

The present study has certain limitations, which should be considered. It should be mentioned that the common practice of dichotomizing health behavior variables may have implications for the findings [23]. Furthermore, this study relied on to selfreports of the different risk behaviors, which may be subject to social desirable answering, and self-reported presence or absence of diabetes. This is a cross-sectional study and only the frequency, and simultaneous clustering of the lifestyle risk factors was reported and no causal claims were made. We would also add that this study was conducted 8-9 years ago since this is the most recent public use data set available. To date, there is no other system regularly producing nationally representative data to monitor and investigate the behavioral risk factors in the Portuguese population. The new National Health Interview Survey is ongoing.

Our results may have important implications in terms of health policy and practice given that the high prevalence of multiple diabetes risk factors underscores the importance of interventions aimed at their reduction. Given that behavioral risk factors such as those investigated in the present study are potentially modifiable, identifying subgroups that are at higher risk of simultaneously displaying multiple factors is of extreme importance if we wish to reduce propensity of the diabetes.

One of the strengths of the present study is that it investigates clusters of diabetes risk factors, in contrast to most other studies, which focus on isolated behaviors. It found that approximately $9 \%$ of the diabetic and $19 \%$ of the non-diabetic individuals, respectively, have multiple risks and identified groups that are generally more at risk. Multiple behavior interventions may not only have a much greater impact on public health than single behavior interventions [24], they may also be more effective and efficient at achieving which goals as well [25].

\section{CONCLUSIONS}

Three major findings can be highlighted. First, the occurrence of the studied risk behaviors in the population is high: $92.1 \%$ of the Portuguese population aged 15 years and over reported at least one risk factor for diabetes. Second, among diabetics, the behavior pattern that indicated a greater increase than that expected at random was the simultaneous occurrence of the three risk factors (smoking, heavy drinking and unhealthy diet). Among nondiabetic individuals the behavior pattern that indicated a greater increase than expected was the simultaneous occurrence of the two risk factors (smoking and heavy drinking). Finally, the most vulnerable groups to the simultaneous occurrence of two or more risk behaviors for diabetes were identified: Men who have 35-44 years, who have secondary education and single or divorced depending diabetic or non-diabetic individuals.

The tendency for risk factors to aggregate has important implications for health promotion, thus, information on subgroups with elevated clustering will help in planning future preventive strategies.

\section{ACKNOWLEDGMENTS}

Luzia Gonçalves has been supported by the PEst-OE/MAT/ UI0006/2014-FCT-Portugal.

\section{REFERENCES}

1. Zimmet P, Alberti KG, Shaw J. Global and societal implications of the diabetes epidemic. Nature 2001;414:782-7.

2. Poortinga $W$. The prevalence and clustering of four major lifestyle risk factors in an English adult population. Prev Med 2007;44:124-8.

3. Moreno-Gómez C, Romaguera-Bosch D, Tauler-Riera P, Bennasar-Veny $M$, Pericas-Beltran J, Martinez-Andreu S, et al. Clustering of lifestyle factors in Spanish university students: the relationship between smoking, alcohol consumption, physical activity and diet quality. Public Health Nutr 2012;15:2131-9.

4. Galán I, Rodríguez-Artalejo F, Tobías A, Díez-Gañán L, Gandarillas A, Zorrilla B. Clustering of behavior-related risk factors and its association with subjective health. Gac Sanit 2005;19:370-8.

5. World Health Organization. Global Health Risks: Mortality and Burden of Disease Attributable to Selected Major Risks. Geneva: World Health Organization; 2009.

6. Matias Dias C, Briz T. Distribuição conjunta de determinantes de saúde relacionados com comportamentos na população portuguesa. Observações Bol Epidemiol INSA 2012;1:4-5.

7. Pronk NP, Anderson LH, Crain AL, Martinson BC, O'Connor PJ, Sherwood NE, et al. Meeting recommendations for multiple healthy lifestyle factors. Prevalence, clustering, and predictors among adolescent, adult, and senior health plan members. Am J Prev Med 2004; $27: 25-33$

8. Chiolero A, Wietlisbach V, Ruffieux C, Paccaud F, Cornuz J. Clustering of risk behaviors with cigarette consumption: A population-based survey. Prev Med 2006;42:348-53.

9. Slattery ML, Potter JD. Physical activity and colon cancer: confounding or interaction? Med Sci Sports Exerc 2002;34:913-9.

10. Schuit AJ, van Loon AJ, Tijhuis M, Ocké M. Clustering of lifestyle risk factors in a general adult population. Prev Med 2002;35:219-24.

11. Word Health Organization. The Word Health Report: Reducing Risks, Promoting Healthy Life. Geneva: World Health Organization; 2002.

12. Mokdad AH, Marks JS, Stroup DF, Gerberding JL. Actual causes of death in the United States, 2000. JAMA 2004;291:1238-45.

13. National Health Institute Doutor Ricardo Jorge. National Statistical Institute. 2005/2006 Portuguese National Health Interview Survey. Lisbon: INSA; 2009.

14. Centers for Disease Control and Prevention. Alcohol and Public Health. Atlanta, GA: Department of Health and Human Services; 2013.

15. Aguiar P, Neto D, Lambaz R, Chick J, Ferrinho P. Prognostic factors during outpatient treatment for alcohol dependence: cohort study with 6 months of treatment follow-up. Alcohol Alcohol 2012;47:702-10.

16. Marques-Vidal P, Dias CM. Trends and Portugal: Results from the national health surveys 1995 to 1996 and 1998 to 1999. Alcohol Clin Exp Res 2005;29:89-97.

17. Centers for Disease Control and Prevention. UK Physical Activity Guidelines. Atlanta, GA: Department of Health and Human Services; 2011.

18. World Health Organization. Global Recommendations on Physical Activity for Health. Geneva: World Health Organization; 2010.

19. Costa E, Oliveira L, Gonçalves L, Dias CM. Dietary patterns of the Portuguese population with and without self-reported diabetes: Data from the fourth National Health Interview Survey. Br J Nutr (unpublished article, under review process). 
20. Silva DA, Peres KG, Boing AF González-Chica DA, Peres MA. Clustering of risk behaviors for chronic noncommunicable diseases: a population-based study in southern Brazil. Prev Med 2013;56:20-4.

21. Fornari C, Donfrancesco C, Riva MA, Palmieri L, Panico S, Vanuzzo D, et al. Social status and cardiovascular disease: a Mediterranean case. Results from the Italian Progetto CUORE cohort study. BMC Public Health 2010;10:574.

22. Fine LJ, Philogene GS, Gramling R, Coups EJ, Sinha S. Prevalence of multiple chronic disease risk factors 2001 National Health Interview Survey. Am J Prev Med 2004;27:18-24.

23. MacCallum RC, Zhang S, Preacher KJ, Rucker DD. On the practice of dichotomization of quantitative variables. Psychol Methods 2002; 7:19-40.

24. Nigg CR, Allegrante JP, Ory M. Theory-comparison and multiple- behavior research: common themes advancing health behavior research. Health Educ Res 2002;17:670-9.

25. Atkins D, Clancy C. Multiple risk factors interventions. Are we up to the challenge? Am J Prev Med 2004;27:102-3.

(c) GESDAV; licensee GESDAV. This is an open access article licensed under the terms of the Creative Commons Attribution Non-Commercial License (http://creativecommons.org/licenses/by-nc/3.0/) which permits unrestricted, non-commercial use, distribution and reproduction in any medium, provided the work is properly cited.

Source of Support: Nil, Conflict of Interest: None declared. 